DOI: https://doi.org/10.11144/Javeriana.upsy16-3.pohm

\title{
Perfiles de orientación hacia la meta y su relación con indicadores de actividad físico- deportiva
}

Goal Orientation Profiles and Their Relation with Indicators of Physical Activity

Recepción: 08 Julio 2014 | Aprobación: 27 Febrero 2017

\author{
Manuel J. De la Torre-Cruz \\ Universidad de Jaén, España \\ Alberto Ruiz-Ariza \\ Universidad de Jaén, España \\ SARA OcAÑA-ExPÓSITO \\ Universidad de Jaén, España \\ EMilio J. MARTínez-López \\ Universidad de Jaén, España \\ ORCID: http://orcid.org/0000-0001-9412-5207
}

a Autor de correspondencia. Correo electrónico: emilioml@ujaen.es

Para citar este artículo: De la Torre-Cruz, M. J., RuizAriza, A., Ocaña-Expósito, S., \& Martínez-López, E. J. (2017). Perfiles de orientación hacia la meta y su relación con indicadores de actividad físicodeportiva. Universitas Psychologica, 16(3), 1-12. https://doi.org/ 10.11144/Javeriana.upsy16-3.pohm

\section{RESUMEN}

El presente estudio examinó la motivación hacia la actividad física empleando la teoría de la orientación hacia la meta. Un total de 786 estudiantes participaron en este estudio. Un procedimiento de análisis de cluster jerárquico permitió identificar cuatro perfiles de orientación hacia la meta. El perfil más adaptativo (alta tarea/alto ego/altas expectativas) se asoció con la obtención de los mejores resultados. Se concluye que los jóvenes con una alta motivación hacia la tarea, alto ego y expectativas de ejecución obtienen las mayores muestras de apoyo por parte de familiares e iguales para la práctica de actividad física se perciben más autoeficaces para realizar dicha actividad, se atribuyen una mejor forma física autopercibida y manifiestan una práctica más frecuente de actividad físico-deportiva.

\section{Palabras clave}

orientación a meta; expectativas de ejecución; autoeficacia; cluster; actividad física

\begin{abstract}
This paper researches motivation for physical activity in the framework of the goal orientation theory. This work is based on data from 786 students. Hierarchical cluster analysis allowed identifying four goal orientation profiles. The most adaptive profile (high task, high ego, high expectancy) is associated with the best results. Students with high motivation for physical activity, ego, and performance expectancy are concluded to obtain more indications of support from their relatives and peers for physical activity practice, perceive themselves self-effective to complete this activity, claim their better self-perceived fitness, and show more frequent physical-sports activity.

Keywords

goal orientation; performance expectancy; self-efficacy; cluster; physical activity
\end{abstract}


La práctica de actividad física en niños y adolescentes es asunto de interés en el ámbito educativo y de la salud. El incremento del sobrepeso y la obesidad infanto-juvenil y sus problemas asociados (diabetes, hipertensión, problemas cardiovasculares, baja autoestima, depresión, rechazo social,...), han promovido el examen de los factores personales, familiares y ambientales que condicionan las actitudes y participación de los jóvenes en actividades físicodeportivas (Baskin et al., 2013; Hyde, Maher, \& Elavsky, 2013; Laird, Fawkner, Kelly, McNamee, \& Niven, 2016; Monshouwer, Have, van Poppel, Kemper, \& Vollenbergh, 2013; Yao \& Rhodes, 2015).

Entre los factores de naturaleza personal que determinan la práctica de actividad física, se encuentra la orientación hacia la meta adoptada por el individuo (Barkoukis, Ntoumanis, \& Thogersen-Ntoumani, 2010; Castillo et al., 2010; Cuevas, García-Calvo, \& Contreras, 2013; Duda \& Nicholls, 1992; Grastén \& Watt, 2016; Liukkonen, Barkoukis, Watt, \& Jaakkola, 2010; Nicholls, 1984). Dos tipos de orientación hacia la meta, tarea y ego, han sido identificadas al respecto. La orientación hacia la tarea se asocia con la adquisición, mejora o perfección de una habilidad. La orientación hacia el ego se concreta en la comparación normativa y en el intento de demostrar superioridad frente a los demás.

La orientación hacia la tarea se asocia positivamente con la motivación intrínseca, la diversión y el disfrute con la práctica de actividad físico-deportiva (Biddle, Wang, Kavussanu, \& Spray, 2003). Por su parte, la orientación hacia el ego se corresponde con patrones motivacionales menos adaptados como la apatía, el aburrimiento y la preocupación por el estatus social en el seno del grupo (Duda \& Nicholls, 1992).

Nicholls (1989) planteó que ambas orientaciones a meta eran ortogonales. Partiendo de esta consideración, Fox, Goudas, Biddle, Duda y Armstrong (1994) sugirieron que una representación más certera de la orientación a meta y las consecuencias asociadas podría extraerse del análisis de los cuatro perfiles obtenidos mediante la combinación de altas y bajas puntuaciones en ambas dimensiones. Sin embargo, una de las limitaciones de este procedimiento reside en el criterio poco exigente de recurrir al valor de la media o mediana cuando se constata que el valor arrojado por la dimensión tarea suele ser muy elevado (Carr, 2012; Standage \& Treasure, 2002). Así pues, la configuración de perfiles empleando otros criterios (grupos extremos o análisis de clúster) podría ofrecer una explicación más próxima a la realidad de la influencia de estas orientaciones a meta (Martínez-Galindo, Alonso, Cervelló, \& Moreno, 2009; Wang, Chatzisarantis, Spray, \& Biddle, 2002; Wang, Liu, Sun, Lim, \& Chatzisarantis, 2010).

En el establecimiento de tales clústeres, se ha recurrido a variables motivacionales derivadas de la teoría de la orientación hacia la meta de Nicholls (Martínez-Galindo et al., 2009; Wang et al., 2002; Wang, Biddle, \& Elliot, 2007; Wang et al., 2010) o bien a las dimensiones incluidas en la teoría de la motivación autodeterminada (Çaglar \& Asçi, 2010; Hein \& Jõessar, 2015; Jaakkola, Wang, Soini, \& Liukkonen, 2015; MorenoMurcia, Cervelló-Gimeno, Huéscar-Hernández, Belando-Pedreño, \& Rodríguez-Marín, 2013; Wang, Morin, Ryan, \& Liu, 2016).

Como se ha indicado, la orientación hacia el ego parece ser menos adaptativa que una orientación hacia la tarea, particularmente, en las situaciones en las que el individuo pone en duda su propia capacidad. En estas circunstancias, tiene lugar la aparición de conductas de logro negativas tales como un nivel de ejecución inferior al esperado, una disminución en el esfuerzo invertido o una falta de persistencia ante la presencia de obstáculos y dificultades (Duda \& Nicholls, 1992; Stavrou, Psychountaky, Georgiadis, Karteroliotis, \& Zervas, 2015). No obstante, Wang et al. (2002) plantearon la posibilidad de que la orientación hacia el ego, pero no la orientación hacia la meta, interactuase con la percepción de competencia de un individuo. Dado el afán comparativo de quienes están orientados hacia el ego, cabe suponer que el esfuerzo y persistencia dedicados serán elevados cuando se perciban a sí mismos competentes, y no así cuando se atribuyan poca 
capacidad. De otro lado, las personas orientadas hacia la tarea no se verán tan influidas por la atribución de capacidad debido que sus esfuerzos se destinan a mejorar su nivel de aprendizaje.

El estudio realizado por Wang et al. (2002), con una muestra de adolescentes, empleó un análisis de clúster jerárquico, tomando como variables de agrupación la orientación hacia la tarea, el ego y la competencia percibida, revelando la existencia de tres grupos: baja, moderada y alta motivación. El grupo altamente motivado (elevada puntuación en tarea, ego y competencia percibida) obtuvo los mayores índices de actividad física y motivación autodeterminada. Los resultados más negativos se observaron en el grupo poco motivado (baja orientación hacia la tarea, el ego y competencia percibida).

Recientemente, Wang et al. (2010), en cuyo estudio emplearon las mismas variables de agrupación que en el conducido por Wang et al. (2002), identificaron cuatro perfiles (alta tarea/alto ego/alta competencia percibida; moderada-alta tarea/moderado-alto ego/elevada competencia percibida; moderadabaja tarea/bajo ego/muy baja competencia percibida y muy baja tarea/muy bajo ego/ baja competencia percibida). El propósito era conocer si los perfiles arrojaban diferencias en la regulación motivacional (intrínseca, identificada, introyectada y externa), actitudes hacia la disciplina de la educación física y frecuencia de participación en actividades físicodeportivas. Los resultados revelaron que los participantes incluidos en los dos primeros grupos lograron los niveles más adaptativos de regulación motivacional, mostraron actitudes más favorables hacia la educación física e informaron de una mayor cantidad de práctica de actividad física.

Con base en lo anterior, el presente estudio analizó la relación existente entre perfiles motivacionales conformados por la orientación hacia la meta y las expectativas de ejecución y diversos indicadores asociados a la práctica de actividad física (motivación intrínseca, utilidad, autoeficacia, apoyo social y familiar, condición física y frecuencia de actividad física semanal) en jóvenes de 10-16 años. Se comprobó, asimismo, si se reproducía la estructura de agrupación (clústeres) obtenida en investigaciones previas, considerando las diferencias culturales de los participantes (asiáticos y anglosajones en los anteriores y españoles en el presente caso). Se hipotetizó que las variaciones observadas en estos perfiles de orientación hacia la meta se asociarían de forma diferencial con la condición física, autoeficacia, apoyo percibido y frecuencia de práctica semanal.

\section{Método}

\section{Participantes}

En este estudio, tomaron parte 786 estudiantes (394 varones, $50.06 \%$ ) que pertenecían a 11 centros educativos (cinco de primaria, cinco de secundaria y uno mixto) de la Comunidad Autónoma de Comunidad Autónoma de Andalucía (omitido para no identificación). De ellos 449 (57.1\%) pertenecían a los dos últimos

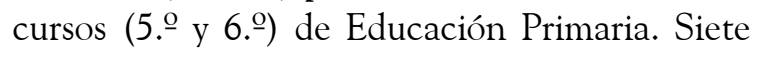
de las instituciones educativas correspondían a poblaciones urbanas ( $>10000$ habitantes) y el resto a zonas rurales ( $<10000$ habitantes). Ninguno de los centros contactados se rehusó a colaborar. El promedio de edad del alumnado fue de 12.04 años $(D E=1.39)$, dentro de un rango comprendido entre los 10 y 16 años. Asimismo, el índice de masa corporal promedio para el conjunto muestral alcanzó un valor de 19.47 (DE $=8.16)$.

Atendiendo al máximo nivel educativo alcanzado por parte de las madres y los padres de los participantes, se obtuvieron los siguientes porcentajes. Respecto a las primeras, $3.94 \%$ carecía de estudios, $24.18 \%$ tenía estudios primarios, $48.22 \%$ estudios de formación profesional o bachillerato y $19.72 \%$ estaba en posesión de un título universitario. No se obtuvo esta información para 31 de las madres (3.94 $\%)$. En relación con el padre, los porcentajes hallados fueron los siguientes: sin estudios (4.07 $\%)$, primarios (20.36\%), secundarios (46.69\%) y universitarios $(22.65 \%)$. No se obtuvo esta 
información para un total de 49 padres (6.23\%). En el momento de la aplicación de las pruebas el $70.74 \%$ de las madres se encontraba en una situación laboral activa (a tiempo parcial o completo), mientras que este porcentaje se incrementó hasta el $88.8 \%$ en el caso de los padres.

\section{Instrumentos}

\section{Cuestionario sociodemográfico}

Se solicitó, entre otra, información sobre el sexo y la edad del participante.

Task and Ego Orientation in Sport Questionnaire ([TEOSQ]; Duda \& Nicholls, 1992) Adaptación española realizada por Balaguer, Castillo y Tomás (1996) que consta de 13 ítems distribuidos en dos dimensiones. La primera, siete ítems, representa una meta orientada hacia la tarea ( $\mathrm{p}$. ej., "Sentiría que he logrado el éxito si he trabajado y me he esforzado"). La segunda, seis ítems, se corresponde con una meta orientada hacia el ego (p. ej., "Sentiría que he logrado el éxito si lo hago mejor que mis compañeros"). Se registra el grado de acuerdo con cada afirmación en una escala Likert de cinco puntos. Para cada dimensión se calcula un valor promedio. Una mayor puntuación equivale a una mayor orientación hacia la tarea o ego, respectivamente.

Expectancy-Value Questionnaire ([EVQ]; Zhu, Su, Chen, Eु Ennis, 2012)

El Cuestionario de expectativa-valor orientado a la educación física consta de 11 ítems agrupados en cuatro subescalas: expectativas de ejecución, importancia, motivación intrínseca y utilidad de la disciplina. Solo se empleó el factor expectativas de ejecución compuesto por cinco ítems (p. ej., "iCuál consideras que es tu nivel de ejecución en las clases de Educación Física?") como un indicador de competencia percibida. El formato de respuesta presenta una escala tipo Likert de cinco puntos. Se obtiene un único valor promediando las respuestas a los cinco ítems.
Una elevada puntuación equivale a una mayor atribución de habilidad y destreza en educación física.

Self-perceived Fitness ([SPF]; Rahmani-Nia, Damitchi, Azizi, $\mathcal{G}$ Hoseini, 2011)

En la Escala de condición física autopercibida se emplearon cuatro ítems (forma física general, fuerza física, resistencia física y flexibilidad) del instrumento elaborado por Ramani-Nia et al. (2011). Se puntúa a lo largo de una escala Likert de 13 puntos. El valor de uno se corresponde con una percepción muy deficiente de cada una de las dimensiones señaladas, mientras que el valor de 13 equivale a una percepción excelente. La condición física atribuida se calculó promediando el valor asignado a cada una de las cuatro dimensiones. Una mayor puntuación equivale a una mejor condición física autopercibida.

\section{Barriers Self-Efficacy (Dishman et al., 2010)}

El Cuestionario de obstáculos de autoeficacia consta de siete ítems que registran a lo largo de una escala Likert de cinco puntos la confianza depositada en la propia capacidad para ser físicamente activo, a pesar de la existencia de circunstancias adversas (clima, tarea escolar, otras actividades). Un ejemplo de ítem es el siguiente: "La mayoría de los días soy capaz de estar físicamente activo durante mi tiempo libre, aunque tenga mucha tarea escolar". Se obtiene una única puntuación equivalente al valor promedio de las respuestas emitidas. Una mayor puntuación refleja una autoeficacia percibida más elevada.

Social Support-Friends and Social Support-Family (Sallis, Taylor, Dowda, Freedson, $\mathcal{E}$ Pate, 2002).

Escala de apoyo social de amigos y Escala de apoyo social familiar miden el apoyo social para la práctica de actividad física en una escala Likert de cinco puntos. Se registra la frecuencia, desde nunca hasta todos los días, con la que 
los jóvenes cuentan con el apoyo de amigos y familiares para realizar actividad física. Tres de los ítems se circunscriben a la ayuda aportada por los iguales (p. ej., "Tus amigos/as te animan para que realices actividades físico-deportivas") y, los cuatro restantes, a la ayuda mostrada por los familiares (p. ej., "Alguien de tu familia fue a verte participar en actividades físico-deportivas"). Se obtiene una puntuación promedio para cada factor. Un valor más elevado refleja un mayor grado de apoyo recibido.

Moderate to Vigorous Physical Activity ([MVPA]; Prochaska, Sallis, EG Long, 2001)

La medida de Actividad física de moderada a vigorosa registra el número de días (desde cero hasta siete) durante la última semana y una semana típica en los que el participante realizó al menos 60 minutos de actividad física de intensidad moderada o vigorosa. La actividad física se define como 'cualquier actividad que aumenta la frecuencia cardíaca y hace que la respiración se acelere'. Se obtiene un valor que equivale al número de días de práctica semanal promediando la respuesta a ambos ítems.

\section{Procedimiento}

Una descripción verbal y escrita de la naturaleza y el propósito del estudio fue dada a los adolescentes, padres y tutores legales, los cuales dieron su consentimiento. Se contó también con la autorización de los directores de los centros y profesores de educación física. El estudio fue aprobado por la Comisión de Bioética de la Universidad de XXX (omitido para no identificación). En el diseño se han tenido en cuenta la normativa legal vigente española que regula la investigación clínica en humanos (Real Decreto 561/1993 sobre ensayos clínicos), la ley de protección de datos de carácter personal (Ley Orgánica 15/1999) y los principios fundamentales establecidos en la Declaración de Helsinki (revisión de 2008).

Las diferentes pruebas se aplicaron en el aula durante alguna de las horas lectivas asignadas a la materia escolar de Educación Física. La participación fue voluntaria y se informó al alumnado de la confidencialidad de sus respuestas. El investigador controlaba el ritmo de cumplimentación de los instrumentos leyendo en voz alta las instrucciones, los enunciados y concediendo un tiempo prudencial para que los chicos y chicas seleccionasen la opción deseada. A su vez, cualquier duda o cuestión planteada era aclarada o resuelta para el conjunto de participantes. El tiempo destinado a completar los instrumentos de medida fue de 45 minutos.

\section{Análisis estadístico}

Los parámetros se muestran como media y desviación estándar. Las diferencias entre sexo se analizaron mediante la prueba t de Student para variables continuas y el test $\chi^{2}$ para variables categóricas. Para conocer la relación entre variables se empleó el coeficiente de correlación de Pearson. El análisis de clúster es una técnica que intenta agrupar variables con objeto de lograr la máxima homogeneidad intragrupo y la mayor diferencia entre grupos. Para determinar los grupos o perfiles, ambos tipos de orientación a meta -tarea y ego- así como las expectativas de ejecución en la clase de educación física -indicador de competencia futura percibida-, se tomaron como variables de agrupación. Previamente a la realización de este análisis, dichas variables fueron tipificadas $(M=0, D E=1)$. Tomando como referente la investigación precedente (Wang et al., 2010) y la imagen del dendograma, se identificaron cuatro grupos empleando el procedimiento de la distancia euclídea al cuadrado. Las puntuaciones típicas próximas o superiores a \pm 0.5 se emplearon como criterio para describir si una variable de perfil es relativamente alta o baja en comparación con lo observado para otro clúster, mientras que las puntuaciones típicas cercanas a \pm 0.3 fueron consideradas como moderadas. Se optó por el empleo de un análisis jerárquico.

Para examinar la existencia de diferencias entre los grupos en las medidas dependientes (condición física percibida, autoeficacia para la 
práctica físico-deportiva, apoyo para la práctica físico-deportiva y frecuencia de actividad semanal), se realizó un análisis multivariado de varianza (MANOVA). Se analizó la posible existencia de diferencias en el porcentaje de chicas y chicos en los distintos clústeres. No se observaron diferencias significativas entre los distintos grupos conformados, $\chi^{2}=(3, N=785)$ $=5.23, p>0.05$. Los posteriores análisis no se diferenciaron según el sexo del participante al no apreciarse diferencias en ninguna de las medidas dependientes. Se estableció como criterio un nivel de confianza del $95 \%(\mathrm{p}<0.05)$. Los cálculos se realizaron con el programa estadístico SPSS, v. 19.0 para WINDOWS (SPSS Inc., Chicago).

\section{Resultados}

\section{Estadísticos descriptivos}

Los valores promedio, desviaciones estándar, índices de consistencia interna y correlaciones entre variables se muestran en la Tabla 1 . Todas las variables correlacionaron positivamente entre sí.

\section{TABLA 1}

Estadísticos descriptivos y valor de las correlaciones entre las diferentes variables para la totalidad de participantes

\begin{tabular}{lccccccccccc}
\hline & Media & $D E$ & Alpha & 1 & 2 & 3 & 4 & 5 & 6 & 7 & 8 \\
\hline Orientación a & 4.26 & 0.58 & 0.68 & 1 & & & & & & & \\
Orinntación a & 2.75 & 0.91 & 0.79 & 0.22 & 1 & & & & & & \\
Expectativas & 3.77 & 0.55 & 0.79 & 0.38 & $0.36^{* *}$ & 1 & & & & & \\
Condición & 8.15 & 1.89 & 0.64 & 0.29 & $0.29^{* *}$ & $0.56^{* *}$ & 1 & & & & \\
Autocficacia & 3.56 & 0.81 & 0.7 & 0.35 & $0.19^{* *}$ & $0.42^{* *}$ & $0.35^{* *}$ & 1 & & & \\
Apoyo de & 3.19 & 0.92 & 0.74 & 0.25 & $0.22^{* *}$ & $0.41^{* *}$ & $0.31^{* *}$ & $0.51^{*}$ & 1 & & \\
Apoyo de de & 3.35 & 0.95 & 0.64 & $0.2^{*}$ & $0.21^{* *}$ & $0.39^{* *}$ & $0.34^{* *}$ & $0.55^{* *}$ & 0.51 & 1 & \\
A. F. semanal & 3.68 & 1.66 & 0.74 & 0.31 & $0.23^{* *}$ & $0.43^{* *}$ & $0.41^{* *}$ & $0.51^{* *}$ & 0.41 & $0.44^{*}$ & 1 \\
\hline
\end{tabular}

Notas. E.F. (Educación Física); A.F.

(Actividad Física); ** $p<0.01$

Fuente: elaboración propia.

Análisis de perfiles

Las puntuaciones medias, desviaciones estándar y puntuaciones $z$ de los diferentes grupos junto a la representación gráfica de los perfiles hallados se muestra en la Tabla 2 y Figura 1, respectivamente.

TABLA 2

Valores promedio, desviaciones estándar y puntuaciones típicas (z) obtenidos en los diferentes perfiles

\begin{tabular}{|c|c|c|c|c|c|c|c|c|c|c|c|c|}
\hline & \multirow{2}{*}{\multicolumn{3}{|c|}{ Clúster 1}} & & \multicolumn{3}{|c|}{ Clüster 3} & \multicolumn{3}{|c|}{ Clusster 4} \\
\hline & & & \multicolumn{3}{|c|}{$(n=256)$} & \multicolumn{3}{|c|}{$(n=206)$} & \multicolumn{3}{|c|}{$(n=146)$} \\
\hline & $M$ & $D E$ & $z$ & $M$ & $D E$ & $z$ & $M$ & $D E$ & $z$ & $M$ & $D E$ & $z$ \\
\hline Variables & & & & & & & & & & & & \\
\hline 1.- Tarea & 3.67 & 0.51 & -0.87 & 4.68 & 0.26 & 0.74 & 4.57 & 0.33 & 0.56 & 3.81 & 0.35 & -0.65 \\
\hline 2. $\mathrm{Ego}$ & 2.22 & 0.73 & .0 .58 & 3.53 & 0.52 & 0.93 & 1.95 & 0.43 & -0.89 & 3.18 & 0.73 & 0.53 \\
\hline 3.- Expectativas & 3.13 & 0.32 & -1.1 & 4.06 & 0.51 & 0.54 & 3.94 & 0.32 & 0.13 & 3.77 & 0.55 & 0.33 \\
\hline
\end{tabular}

Fuente: elaboración propia.

El primer clúster estaba compuesto por estudiantes con puntuaciones muy bajas en todas las variables. Este grupo quedó conformado por 178 participantes (97 chicas). El segundo clúster se caracterizó por arrojar puntuaciones elevadas en orientación hacia la tarea, el ego y expectativas de ejecución y quedó conformado por 256 estudiantes (136 varones). El tercer clúster agrupó un total de 206 alumnos (111 chicos); su perfil fue de elevadas puntuaciones en orientación hacia la tarea, muy bajas puntuaciones en ego y unas expectativas de ejecución moderadas. El cuarto clúster reveló un perfil caracterizado por puntuaciones muy bajas en orientación hacia la tarea, elevadas en ego y moderadas en expectativas de ejecución. Este último grupo congregó a 146 participantes (80 chicas). 
Figura 1.

Perfiles de orientación a meta y expectativas de ejecución para la solución de cuatro grupos.

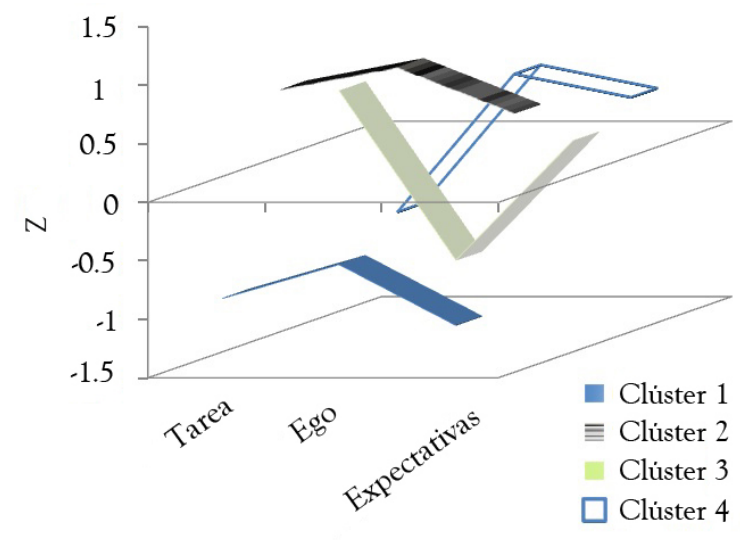

Fuente elaboración propia.

Diferencias en condición física percibida, autoeficacia para la práctica físico-deportiva, apoyo para la práctica físico-deportiva y práctica física autoinformada en función de los grupos

El análisis multivariado de varianza (MANOVA) arrojó un efecto principal significativo de la variable grupo, $\lambda=0.69, F_{(15,2148.12)}=20.16$, $p>0, \eta=0.11$. Posteriores análisis univariados de varianza revelaron la existencia de diferencias para la totalidad de medidas dependientes (Tabla 3).

\section{TABLA 3}

Comparación de las puntuaciones promedio (desviaciones estándar entre paréntesis) en las diferentes medidas dependientes en función de los grupos

\begin{tabular}{lcccccccc} 
& Clúster & Clúster & Clúster & Clúster & $F(3$, & & \\
& 2 & 3 & 4 & 1 & $782)$ & $p$ & $\eta$ & Post-hoc \\
\hline Condición fisica & 8.89 & 8.2 & 8.51 & 6.76 & 56.24 & 0 & 0.18 & $2>3$ y 1,4 y $3>1$ \\
& $(1.77)$ & $(1.72)$ & $(1.79)$ & $(1.57)$ & & & & \\
Autoeficacia & 3.82 & 3.7 & 3.62 & 2.98 & 48.93 & 0 & 0.16 & 2,3 y $4>1$ \\
& $(0.77)$ & $(0.68)$ & $(0.78)$ & $(0.75)$ & & & & \\
Apoyo iguales & 3.53 & 3.17 & 3.29 & 2.65 & 36.82 & 0 & 0.12 & $2>3$ y 1,4 y $3>1$ \\
& $(0.9)$ & $(0.83)$ & $(0.89)$ & $(0.81)$ & & & & \\
Apoyo familia & 3.72 & 3.41 & 3.4 & 2.73 & 45.74 & 0 & 0.15 & $2>3$ y $4>1$ \\
& $(0.88)$ & $(0.83)$ & $(0.86)$ & $(0.93)$ & & & & \\
Actividad & & & & & & \\
semanal & 4.36 & 3.84 & 3.71 & 2.53 & 52.39 & 0 & 0.17 & $2>3$ y $4>1$ \\
& $(1.51)$ & $(1.51)$ & $(1.54)$ & $(1.5)$ & & & & \\
\hline
\end{tabular}

Fuente: elaboración propia.

Comparaciones a posteriori empleando la corrección de Bonferroni (0.05/6 = 0.008), mostraron que el grupo con el perfil motivacional más desadaptado (G-1) obtuvo el menor grado de autoeficacia para la práctica de actividad físicodeportiva respecto a los restantes.

Asimismo, los participantes del clúster con elevadas puntuaciones en todas las dimensiones (G-2) se atribuyeron una mejor condición física y expresaron sentirse más apoyados por los iguales, en comparación con los grupos en los que los estudiantes coincidían en mostrar una muy baja orientación hacia el ego (G-3 y G-1). Además, los grupos en cuyo perfil se halló una puntuación elevada en cualquiera de las orientaciones a meta (G-4 y G-3) diferían en estas medidas respecto al grupo motivacionalmente más desadaptado (G-1).

Por último, se observaron diferencias en el apoyo percibido en la familia y la frecuencia de actividad física autoinformada. Específicamente, el grupo con el perfil de puntuaciones más elevadas (G-2) logró mejores resultados que aquellos otros cuya orientación, bien hacia la tarea o bien hacia el ego, fue baja (G-3 y G-4), quienes, a su vez, puntuaron más en las citadas medidas que los estudiantes del grupo de bajo perfil general (G-1).

\section{Discusión}

El presente estudio analizó la existencia de diferencias en diversos indicadores asociados a la actividad física en función del perfil de orientación a meta y expectativas de ejecución exhibido por alumnos de educación primaria y secundaria. Se examinó, además, si los grupos establecidos mostraban un perfil semejante al observado en estudios previos, así como la posible existencia de diferencias en el porcentaje de chicas y chicos incluidos en cada uno de los grupos. El análisis de clúster determinó la existencia de cuatro perfiles: muy baja tarea/muy bajo ego/muy bajas expectativas de ejecución (Clúster 1); alta tarea/alto ego/altas expectativas de ejecución (Clúster 2); alta tarea/bajo ego/ moderadas expectativas de ejecución (Clúster 3) y baja tarea/alto ego/moderadas expectativas de ejecución (Clúster 4). Los grupos más extremos (Clúster 1 y 2 ) coinciden en su totalidad 
con dos de los obtenidos en los estudios de Wang et al. (2002 y 2010). Sin embargo, dos de los conglomerados parecen ser específicos para esta muestra de estudio (Clúster 3 y 4, respectivamente).

Si bien en los grupos uno y cuatro, el porcentaje de chicas fue superior al de chicos, no se observaron diferencias en cuanto al sexo del participante en ninguno de los cuatro grupos considerados. La distribución de participantes en cada uno de los grupos resultó bastante homogénea $(22.62 \%, 32.61 \%, 26.24 \%$ y 18.48 $\%$, respectivamente, para los grupos $1,2,3$ y 4).

Nicholls (1989) abogó por la ortogonalidad e independencia de las dos orientaciones a meta. De hecho, la investigación realizada con adolescentes anglosajones así lo corroboró (Standage \& Treasure, 2002; Wang et al., 2002). Sin embargo, en nuestro estudio se observó una cierta relación entre ambas dimensiones. Este resultado coincide con el obtenido por Wang et al. (2010), realizado con adolescentes asiáticos y con el de Sicilia, Moreno y Rojas (2008) en una muestra de estudiantes españoles. La falta de independencia entre las dos orientaciones hacia la meta puede implicar que los niños y adolescentes de nuestro estudio no utilizan un único criterio a la hora de valorar su posibilidad de éxito. De esta forma, la consecución de un determinado logro puede estar condicionada tanto por una percepción de progreso personal, así como por la obtención de un mejor resultado respecto a un rival.

De otro lado, el grupo con valores más elevados en las variables de agrupación (alta tarea/alto ego/altas expectativas) obtuvo los mejores resultados al atribuirse una mejor condición física, sentirse más autoeficaces para practicar actividad físico-deportiva a pesar de la existencia de circunstancias adversas, recibir las mayores muestras de apoyo por parte de familiares e iguales para realizar dicha práctica e informar de la realización de más actividad física semanal. En varios de los indicadores (apoyo de iguales y familiares, condición física atribuida y frecuencia de actividad física autoinformada), los resultados diferían en relación con el grupo que mostraba una elevada orientación a la tarea, muy baja orientación al ego y un nivel moderado de expectativas de ejecución. De otro lado, las valoraciones más bajas en todas las medidas registradas se sucedieron en el grupo que presentó un perfil motivacional más desadaptado (baja tarea/bajo ego/bajas expectativas).

Resultados muy similares han sido obtenidos en otras investigaciones. Por ejemplo, Wang et al. (2002) obtuvieron que el clúster etiquetado "altamente motivado" desplegó el máximo nivel de actividad física autoinformada, logró el mayor índice de motivación autodeterminada y concibió a la habilidad deportiva como una cualidad susceptible de mejora mediante la práctica y el esfuerzo. De otro lado, el grupo "poco motivado" presentó las menores puntuaciones en estos indicadores. En sintonía con los anteriores, Standage y Treasure (2002) hallaron que los jóvenes que se atribuían una elevada orientación tanto a la tarea como al ego informaron de mayores índices de motivación intrínseca y regulación identificada hacia la educación física que cualquiera de los grupos de comparación que mostraban un bajo perfil de orientación hacia la tarea.

Del conjunto de estos resultados se desprenden dos conclusiones. En primer lugar, la menor puntuación alcanzada en todos los indicadores motivacionales o de actividad físicodeportiva hace que los niños y jóvenes incluidos en cualquiera de los clústeres con un pobre perfil de orientación a meta se convierta en un grupo de riesgo. En segundo lugar, que la pertenencia a un grupo con elevados valores en orientación a meta y ego puede responder en términos motivacionales a la adopción de un perfil ideal, aunque es cierto que aquellos participantes altamente orientados hacia la tarea y moderadamente hacia el ego también suelen presentar resultados positivos en algunas de las dimensiones citadas.

De forma un tanto sorprendente, no se hallaron diferencias entre los grupos en los que la combinación orientación a tarea y orientación a ego mostraba un perfil opuesto entre sí (G-3 y G-4), cuando se intuye que los alumnos con una mayor orientación hacia la tarea presentan un patrón motivacional más adaptativo. Una posible 
explicación de esta ausencia de diferencias puede deberse a la tercera variable considerada, expectativas de ejecución. De hecho, el examen de los perfiles de ambos grupos reveló que las expectativas de ejecución fueron más elevadas en el grupo de alta orientación al ego. Tal vez la mayor percepción de competencia futura mitigó la obtención de peores resultados en un grupo que, a priori, presenta un perfil motivacional menos adaptativo.

Diferentes autores (Duda \& Nicholls, 1992; Sicilia et al., 2008; Standage \& Treasure, 2002; Wang et al., 2002, Wang et al., 2007; Wang et al., 2010) explican estos resultados recurriendo a la interacción entre orientación al ego y competencia percibida. Esta interpretación sostiene que los individuos orientados hacia la tarea no se ven afectados por su atribución de capacidad personal puesto que su fin es lograr una mejora personal mediante el esfuerzo. Por el contrario, dado que los individuos orientados hacia el ego muestran preocupación por su habilidad en términos normativos, es posible que se sientan motivados, activos y participativos cuando se atribuyan un elevado nivel competencial, pero no así cuando carezcan de este.

Conviene, no obstante, hacer mención de algunas limitaciones de este estudio. En primer lugar, las relativas a los sesgos propios derivados del empleo de las medidas de autoinforme (falsedad, expectativas, halo,...). En segundo lugar, su naturaleza correlacional impide el establecimiento de relaciones causales entre las variables consideras. En tercer lugar, la competencia percibida no ha sido evaluada en su forma habitual, sino a través de una expectativa asociada al rendimiento en las clases de educación física. A pesar de ello, consideramos que ambos constructos tienen un significado similar que tan solo difieren en perspectiva temporal (presente frente a futuro).

Se concluye que los jóvenes con una alta motivación hacia la tarea, ego y expectativas reciben las mayores muestras de apoyo por parte de familiares e iguales para la práctica de actividad física, se perciben más autoeficaces para realizar dicha actividad, se atribuyen una mejor forma física y manifiestan una práctica más frecuente de actividad físicodeportiva. El análisis de perfiles puede posibilitar intervenciones destinadas a estimular una orientación más positiva que redunde en una mayor adherencia hacia la actividad de niños y adolescentes en contextos formales e informales (Moreno-Murcia et al., 2013). A modo de ejemplo, el establecimiento de objetivos a corto plazo basados en la mejora personal, la organización de actividades de distinta naturaleza que permitan la toma de decisiones individuales, la promoción de la autoevaluación empleando como criterio el esfuerzo realizado, la flexibilización de los tiempos de aprendizaje (respetar los ritmos y conceder un tiempo adicional a aquellos que tengan mayor dificultad para el aprendizaje de una habilidad), la emisión de mensajes verbales positivos centrados en la persistencia ante la dificultad, el intercambio de impresiones entre participantes con habilidades heterogéneas y la interpretación del error como oportunidades para aprender y mejorar, son algunas de las actuaciones que podrían incrementar una orientación motivacional centrada en la tarea (Mora, Cruz, \& Sousa, 2013).

Como prospectiva de futuro se plantea la posibilidad de recurrir al empleo de medidas objetivas destinadas a la evaluación de la frecuencia de actividad física (acelerómetros) o rendimiento físico (longitud de salto, velocidad, fuerza muscular, resistencia aeróbica), en aras de establecer la posible relación existente entre estas y los diferentes perfiles de orientación a meta.

\section{Agradecimientos}

El presente estudio ha sido financiado mediante el proyecto de investigación UJA2013/08/29 que patrocina la fundación Caja Rural de Jaén. Este proyecto se enmarca dentro del programa de apoyo a actividades I + D + I de la Universidad de Jaén (R6/8/2013).

Se agradece la colaboración de todos/as los/ las participantes, así como la de los centros educativos y sus responsables, ya que sin su 
tiempo y ayuda no se habría podido realizar este estudio.

\section{Referencias}

Balaguer, I., Castillo, I., \& Tomás, I. (1996). Análisis de las propiedades psicométricas del Cuestionario de Orientación al Ego y a la Tarea en el Deporte (TEOSQ) en su traducción al castellano. Psicológica, 17, 71-81.

Barkoukis, V., Ntoumanis, N., \& ThogersenNtoumani, C. (2010). Developmental changes in achievement motivation and affect in physical education: Growth trajectories and demographic differences. Psychology of Sport and Exercise, 11, 83-90. http://dx.doi.org/10.1016/ j.psychsport.2009.04.008

Baskin, M. L., Thind, H., Affuso, O., Gary, L. C., LaGory, M., \& Hwang, S. (2013). Predictors of moderate-to-vigorous physical activity (MVPA) in African American young adolescents. Annual Behavioral Medicine, 45, S142-S150. http://dx.doi.org/10.1007/ s12160-012-9437-7

Biddle, S. J. H., Wang, C. K. J., Kavussanu, M., \& Spray, C. S. (2003). Correlates of achievement goal orientations in physical activity: A systematic review of research from 1990-2000. European Journal of Sport Sciences, 3(5), 1-20.

Çaglar, E., \& Asçi, F. H. (2010). Motivational cluster profiles of adolescents athletes: An examination of differences in physical-self perception. Journal of Sports Science and Medicine, 9(2), 231-238.

Carr, S. (2012). High task/high ego oriented students' reasons for endorsing task and ego goals in the context of physical education. Applied Psychology: An International Review, 61, 540-563. http://dx.doi.org/10.1111/ j.1464-0597.2012.00505x

Castillo, I., Tomás, I., Balaguer, I., Fonseca, A. M., Dias, C., \& Duda, J. L. (2010). The Task and Ego Orientation in Sport Questionnaire: Testing for measurement invariance and latent mean differences in Spanish and Portuguese adolescents. International Journal of Testing, 10, 21-32. http://dx.doi.org/ 10.1080/15305050903352107

Cuevas, R., García-Calvo, T., \& Contreras, O. (2013). Perfiles motivacionales en educación física: una aproximación desde la teoría de las metas de logro $2 \times 2$. Anales de Psicología, 29, 685-692. http:// dx.doi.org/10.6018/analesps.29.3.175821

Dishman, R. K., Hales, D. P., Sallis, J. F., Saunders, R., Dunn, A. L., Bedimo-Rung, A. L., \& Ring, K. B. (2010). Validity of social-cognitive measures for physical activity in middle-school girls. Journal of Pediatric Psychology, 35, 72-88. http:// dx.doi.org/10.1093/jpepsy/jsp031

Duda, J. L., \& Nicholls, J. G. (1992). Dimensions of achievement motivation in schoolwork and sport. Journal of Educational Psychology, 84(3), 290-299.

Fox, K., Goudas, M., Biddle, S., Duda, J. L., \& Armstrong, N. (1994). Children's task and ego goal profiles in sport. British Journal of Educational Psychology, 64(2), 253-261.

Grastén, A., \& Watt, A. (2016). Perceptions of motivational climate, goal orientation, and light-to-vigorous-intensity physical activity engagement of a simple of Finnish grade 5 to 9 students. International Journal of Exercise Science, 9(3), 291-305.

Hein, V., \& Jõessar, H. (2015). How perceived autonomy support from adults and peer motivational climate are related with selfdeterminated motivation among young athletes. International Journal of Sport an Exercise Psychology, 13, 193-204. http:// dx.doi.org/10.1080/1612197X.2014.947304 Hyde, A. L., Maher, J. P., \& Elavsky, S. (2013). Enhancing our understanding of physical activity and wellbeing with a lifespan perspective. International Journal of Wellbeing, 3, 98-115. http:// dx.doi.org/10.5502/ijw.v3i1.6

Jaakkola, T., Wang, C. K. J., Soini, M., \& Liukkonen, J. (2015). Students 
'perceptions of motivational climate and enjoyment in Finnish physical education: A latent profile analysis. Journal of Sports Science and Medicine, 14(3), 477-483.

Laird, Y., Fawkner, A., Kelly, P., McNamee, L., \& Niven, A. (2016). The role of social support on physical activity behavior in adolescent girls: A systematic review and metaanalysis. International Journal of Behavioral Nutrition and Physical Activity, 13, 79. http:// dx.doi.org/10.1186/s12966-016-0405-7

Liukkonen, J., Watt, A., Barkoukis, V., Watt, A., \& Jaakkola, T. (2010). Motivational climate and students 'emotional experiences and effort in physical education. The Journal of Educational Research, 103, 295-308. http:// dx.doi.org/10.1080/00220670903383044

Martínez-Galindo, C., Alonso, N., Cervelló, E., \& Moreno, J. A. (2009). Perfiles motivacionales y disciplina en clases de educación física. Diferencias según las razones del alumnado para ser disciplina y la percepción del trato generado por el profesorado en el aula. Cultura y Educación, $21(3), 331-343$.

Monshouwer, K., ten Have, M., van Poppel, M., Kemper, H., \& Vollebergh, W. (2013). Possible mechanisms explaining the association between physical activity and mental health: Finding from the 2001 Dutch health behavior in school-aged children survey. Clinical Psychological Science, 1, 67-74. http:// dx.doi.org/10.1177/2167702612450485

Mora, A., Cruz, J., \& Sousa, C. (2013). Cómo mejorar el clima motivacional y los estilos de comunicación en el ámbito de la Educación Física y el deporte. Infancia y Aprendizaje, 36, 91-103. http:// dx.doi.org/10.1174/021037013804826546

Moreno-Murcia, J. A., Cervelló-Gimeno, E., Huéscar-Hernández, E., Belan-do Pedreño, N., \& Rodríguez-Marín, J. (2013). Motivational profiles in physical education and their relation to the Theory of Planned Behavior. Journal of Sports Science and Medicine, 12, 551-558.
Nicholls, J. G. (1984). Achievement motivation: Conceptions of ability, subjective, experience, task choice, and performance. Psychological Review, 91, 328-346.

Nicholls, J. G. (1989). The competitive ethos and democratic education. Cambridge, MA: Harvard University Press.

Prochaska, J. J., Sallis, J. F., \& Long, B. (2001). A physical screening measure for use with adolescents in primary care. Archives in Pediatrics and Adolescence Medicine, 155, 554-559. http://dx.doi.org/10.1001/ archpedi.155.5.554

Rahmani-Nia, F., Damitchi, A., Azizi, M., \& Hoseini, R. (2011).Associations between self-perceived and measured physical fitness of male college students. World Applied Sciences Journal, 14(9), 1331-1338.

Sallis, J. F., Taylor, W. C., Dowda, M., Freedson, P. S., \& Pate, R. R. (2002). Correlates of vigorous physical activity for children in grades 1 through 12: Comparing parentreported and objectively measured physical activity. Pediatric Exercise Science, 14(1), 30-44.

Sicilia, A., Moreno, J. A., \& Rojas, A. J. (2008). Motivational profiles and flow in physical education lessons. Perceptual and Motor Skills, 106, 473-494. http:// dx.doi.org/10.2466/PMS.106.2.473-494

Standage, M., \& Treasure, D. C. (2002). Relationship among achievement goal orientations and multidimensional situational motivation in physical education. British Journal of Educational Psychology, 72, 87-103.

Stavrou, N. A. M., Psychountaky, M., Georgiadis, E., Karteroliotis, K., \& Zervas, Y. (2015). Flow theory-goal orientation theory: Positive experience is related to athlete's goal orientation. Frontiers in Psychology, 6, 1499. https:// dx.doi.org/10.3389/fpsyg.2015.01499

Wang, C. K. J., Biddle, S. J. H., \& Elliot, A. J. (2007). The $2 \times 2$ achievement goal framework in a physical education context. Psychology of Sport and Exercise, 8(2), 147-168. 
Wang, C. K. J., Chatzisarantis, N. L. D., Spray, C. M., \& Biddle, S. J. H. (2002). Achievement goal profiles in school physical education: Differences in self-determination, sport ability beliefs and physical activity. British Journal of Educational Psychology, 72, 433-455.

Wang, C. K. J., Liu, W. C., Sun, Y., Lim, B. S. C., \& Chatzisarantis, N. L. D. (2010). Chinese students' motivation in physical activity: Goal profile analysis using Nicholl 's achievement goal theory. IJSEP, 8, 284-231.

Wang, C. K. J., Morin, A. J. S., Ryan, R. M., \& Liu, W. C. (2016). Students - motivational profiles in the physical education context. Journal of Sport and Exercise Psychology, 38, 1-46. http:// dx.doi.org/10.1123/jsep.2016-0153

Yao, C. A., \& Rhodes, R. E. (2015). Parental correlates in child and adolescent physical activity: A metaanalysis. International Journal of Behavioral Nutrition, 12, 10. http://dx.doi.org/10.1186/ s12966-015-0163-y

Zhu, X., Sun, H., Chen, A., \& Ennis, C. (2012). Measurement invariance of ExpectancyValue Questionnaire in physical education. Measurement in Physical Education and Exercise Science, 16, 41-54. http:// dx.doi.org/10.1080/1091367X.2012.639629

\section{Notas}

* Artículo de investigación. 